\title{
A novel mating system analysis for modes of self-oriented mating applied to diploid and polyploid arctic Easter daisies (Townsendia hookeri)
}

\author{
SL Thompson ${ }^{1}$ and K Ritland ${ }^{2}$ \\ ${ }^{1}$ Department of Botany and Centre for Biodiversity Research, University of British Columbia, Vancouver, British Columbia, Canada V6T \\ 1Z4; ${ }^{2}$ Department of Forest Sciences, University of British Columbia, Vancouver, British Columbia, Canada V6T 1Z4
}

\begin{abstract}
We have developed a new model for mating system analysis, which attempts to distinguish among alternative modes of self-oriented mating within populations. This model jointly estimates the rates of outcrossing, selfing, automixis and apomixis, through the use of information in the family structure given by dominant genetic marker data. The method is presented, its statistical properties evaluated, and is applied to three arctic Easter daisy populations, one consisting of diploids, the other two of tetraploids. The tetraploids are predominantly male sterile and reported to be apomictic while the diploids are male fertile. In each Easter daisy population, 10 maternal arrays of six progeny were assayed for amplified fragment length polymorphism markers. Estimates, confirmed with likelihood ratio tests of mating hypotheses, showed apomixis to be predominant in all populations (ca. $70 \%$ ), but selfing or automixis was
\end{abstract}

moderate (ca. 25\%) in tetraploids. It was difficult to distinguish selfing from automixis, and simulations confirm that with even very large sample sizes, the estimates have a very strong negative statistical correlation, for example, they are not independent. No selfing or automixis was apparent in the diploid population, instead, moderate levels of outcrossing were detected (23\%). Low but significant levels of outcrossing $(2-4 \%)$ seemed to occur in the male-sterile tetraploid populations; this may be due to genotyping error of this level. Overall, this study shows apomixis can be partial, and provides evidence for higher levels of inbreeding in polyploids compared to diploids and for significant levels of apomixis in a diploid plant population.

Heredity (2006) 97, 119-126 doi:10.1038/sj.hdy.6800844; published online 24 May 2006

Keywords: apomixis; automixis; autopolyploidy; mating system estimation; outcrossing; selfing

\section{Introduction}

The mating system governs the transmission of gametes among generations, hence virtually all investigations of the dynamics of genetic change are concerned, directly or indirectly, with the mating process (Clegg, 1980). Traditionally, estimation of mating systems using genetic markers has assumed a mixture of selfing and random outcrossing (Ritland, 2002). This model stems from the conceptual advance of Allard and his coworkers (cf. Brown and Allard, 1970) who demonstrated that plant populations could practice a mixture of mating types. Yet many asexual species continue to be treated as exhibiting one fixed mode of reproduction: either obligate or facultative asexuality. Furthermore, the criteria used to discriminate between these two categories varies from study to study. Finally, selfing and asexuality have rarely been jointly considered as alternative to sexual outcrossing. Mating patterns are best interpreted as a complex continuum, ranging from random outcrossing to selfing to complete asexuality (Bayer et al, 1990).

'Self-oriented mating' is defined here as a transmission bias so that a greater proportion of an organism's

Correspondence: SL Thompson, Département de sciences biologiques, Université de Montréal, CP 6128, succursale centre-ville, Montréal, Quebec, Canada H3C 3JV. E-mail: st@ceythompson.com

Received 2 November 2005; accepted 21 April 2006; published online 24 May 2006 genome is passed to its progeny relative to that expected under random outcrossing. Self-oriented mating systems include the reproductive phenomena of self-fertilization, automixis and apomixis. Self-fertilization (autogamy) is the union of products from different meioses from the same individual, and causes heterozygosity to be theoretically reduced by one-half within progeny for each generation. This differs from automixis (sometimes known as automictic parthenogenesis, reviewed in Mogie, 1986), which is the fusion of two products of the same meiosis. Automixis is known to occur rarely among eggs in animals, among fungal meiospores, and among derivatives of the megasporocyte (egg sac precursor) in plants. Under automixis, heterozygosity is likewise lost among progeny, yet this loss occurs at a slower rate than under selfing, decreasing by one-third with each generation (for alternative alleles ' $A$ ' and ' $a$ ', the probability of sampling, without replacement, an 'Aa' gamete from 'AAaa' tetrad is two-thirds). Automixis has yet to be incorporated into mating system estimation methods. Apomixis (or agamospermy) is parthenogenetic reproduction through seed and can occur via a myriad of embryological pathways (see Nogler, 1984 for authoritative descriptions). Apomixis results in the transmission of an exact copy of the maternal genotype.

Shifts in breeding system may occasionally be facilitated or induced by polyploidy (Richards, 1997). Polyploidy, the possession of more than two complete 
chromosome sets, is a common class of genome change throughout plants and animals (reviewed in Otto and Whitton, 2000). Polyploidy is known to impact selfing ability in some organisms (Stebbins, 1950; Levin, 1983; Cook and Soltis, 2000; but see Mable, 2004), may lead to a breakdown of self-incompatibility (Chawla et al, 1997; Stone, 2002) and may be accompanied by relaxed inbreeding depression (Husband and Schemske, 1997) although increased inbreeding depression may also occur depending on the mode of gene action (Ronfort, 1999). Polyploidy is also associated with asexual reproduction, in fact, $99 \%$ of all known apomictic plants are polyploid but usually for asymmetric or odd ploidy levels (Nogler, 1984; Asker and Jerling, 1992). Owing to its prevalence and potential impact, particularly within the flowering plants (Otto and Whitton, 2000), mating system models must incorporate the additional layer of complexity which polyploidy introduces.

The goal of the present study was to develop a procedure for measuring alternative modes of selforiented mating in diploids and tetraploids for use with dominant molecular markers and to test hypotheses about mating patterns using one diploid and two polyploid populations of the arctic Easter daisy (Townsendia hookeri, Asteraceae) from Canada's Yukon Territory. For each population, progeny arrays were assayed using dominant amplified fragment length polymorphism (AFLP) markers, and following joint estimation of the alternative modes of self-mating, likelihood ratio tests were conducted to test for the presence/absence of mating modes. We expected less self-oriented mating to occur within the diploid population, as inbreeding depression is usually greater in diploids, and also expected apomixis to be mixed with selfing or automixis within polyploid populations, as apomixis is not necessarily a fixed condition in many plant populations (Bayer et al, 1990).

\section{Materials and methods}

\section{A new estimation model for self-oriented matings}

Estimation of mixed mating systems with tetraploidy and dominant markers has not been previously performed. Ritland (1990) presented a program, 'mldt', for the case of diploid dominant markers, whereas Murawski et al (1994) presented a model and program, 'mltet', for tetraploids under codominance (both programs are available at http://www.genetics. forestry.ubc.ca/ritland/programs.html). In addition, estimation of mating systems with potential automixis has never been considered with any type of marker or inheritance mode. Here, we describe the procedure for using dominant markers to jointly estimate outcrossing and the three modes of self-oriented mating (apomixis, automixis and selfing) under either diploid or tetraploid inheritance.

For the sexual modes (ie outcrossing and selfing), with no double reduction in the tetraploid, gametes are sampled from parents without replacement. Double reduction (sampling with replacement) is unlikely as multivalents, a necessity for double reduction, were rarely observed in tetraploid T. hookeri (Beaman, 1957), and double reduction is generally not found in tetraploids (Julier et al, 2003). For the general case of parent genotype $\mathrm{A}_{i} \mathrm{~A}_{j} \mathrm{~A}_{k} \mathrm{~A}_{l}$, where subscripts index alternative alleles, there are six possible gametes, each of equal probability (1/6): $\mathrm{A}_{i} \mathrm{~A}_{j}, \mathrm{~A}_{i} \mathrm{~A}_{k}, \mathrm{~A}_{i} \mathrm{~A}_{l}, \mathrm{~A}_{j} \mathrm{~A}_{k}, \mathrm{~A}_{j} \mathrm{~A}_{l}$ and $\mathrm{A}_{k} \mathrm{~A}_{l}$. The probabilities of progeny gametes at a diallelic locus, conditioned on parent genotype, can be calculated. These are given in Table 1, for both codominance and dominance.

From these gametic probabilities, the basic probabilities of the mating system model (the probabilities of offspring genotypes) are obtained, separately for outcrossing and selfing. These are given in Table 2 for the case of dominance, where $q$ is the frequency of the recessive allele in the population. With selfing, the progeny frequencies are the squares of the gametic phenotype frequencies. With random outcrossing, the progeny frequencies are functions of the pollen pool frequencies of the dominant gamete $\left(1-q^{2}\right)$ and of the recessive gamete, $q^{2}$. These outcrossing probabilities are approximations that ignore the presence of inbred gametes (there are two copies of an allele in each gamete).

To obtain probabilities of progeny for the parthenogenetic modes (ie apomixis and automixis), we assume no double reduction during meiosis, which means the maternal genotype is sampled without replacement. With apomixis, the meiotic product is the complete maternal genotype, for example, the progeny phenotype

Table 1 Probabilities of gametes from autotetraploid parents assuming double reduction, under codominance and dominance, respectively

\begin{tabular}{lccccc}
\hline Gamete & \multicolumn{5}{c}{ Parent genotype } \\
\cline { 2 - 5 } & AAAA & AAAa & AAaa & Aaaa & aaaa \\
\hline Codominance & & $1 / 2$ & $1 / 6$ & 0 & 0 \\
AA & 1 & $1 / 2$ & $2 / 3$ & $1 / 2$ & 0 \\
Aa & 0 & 0 & $1 / 6$ & $1 / 2$ & 1 \\
aa & 0 & & & & \\
Dominance & & 1 & $5 / 6$ & $1 / 2$ & 0 \\
$\quad$ A_ & 1 & 0 & $1 / 6$ & $1 / 2$ & 1 \\
aa & 0 & & & & \\
\hline
\end{tabular}

Table 2 Probabilities of tetraploid offspring genotypes with dominance and assuming double reduction, under outcrossing, selfing, automixis and apomixis, respectively

\begin{tabular}{lccccc}
\hline Offspring genotype & \multicolumn{5}{c}{ Parent genotype } \\
\cline { 2 - 6 } & AAAA & $A A A a$ & AAaa & Aaaa & aaaa \\
\hline $\begin{array}{l}\text { Outcrossing } \\
\text { A__ }\end{array}$ & 1 & 1 & $\left(1-q^{2} / 6\right)$ & $\left(1-q^{2} / 2\right)$ & $1-q^{2}$ \\
aaaa & 0 & 0 & $q^{2} / 6$ & $q^{2} / 2$ & $q^{2}$ \\
Selfing & & & & & \\
A__ & 1 & 1 & $35 / 36$ & $3 / 4$ & 0 \\
aaaa & 0 & 0 & $1 / 36$ & $1 / 4$ & 1 \\
Automixis & & & & & \\
A_ & 1 & 1 & $17 / 18$ & $5 / 6$ & 0 \\
aaaa & 0 & 0 & $1 / 18$ & $1 / 6$ & 1 \\
Apomixis & & & & & \\
A_ & 1 & 1 & 1 & 1 & 0 \\
aaaa & 0 & 0 & 0 & 0 & 1 \\
\hline
\end{tabular}


is identical to the parental phenotype. With automixis the situation is more complicated. There are three equally probable ways that the alleles of the maternal genotype $\mathrm{A}_{i} \mathrm{~A}_{j} \mathrm{~A}_{k} \mathrm{~A}_{l}$, can be distributed among meiotic products: (1) $\mathrm{A}_{i} \mathrm{~A}_{j} / \mathrm{A}_{i} \mathrm{~A}_{j} / \mathrm{A}_{k} \mathrm{~A}_{l} / \mathrm{A}_{k} \mathrm{~A}_{l}$, (2) $\mathrm{A}_{i} \mathrm{~A}_{k} / \mathrm{A}_{i} \mathrm{~A}_{k} / \mathrm{A}_{j} \mathrm{~A}_{l} / \mathrm{A}_{j} \mathrm{~A}_{l}$ and (3) $\mathrm{A}_{i} \mathrm{~A}_{l} / \mathrm{A}_{i} \mathrm{~A}_{l} / \mathrm{A}_{j} \mathrm{~A}_{k} / \mathrm{A}_{j} \mathrm{~A}_{k}$. There are six potential matings within each of these three segregation patterns, leading to 18 possible automictic outcomes. Of these, 12 (or 3/4) will be tetraallelic (same as the maternal genotype), whereas the remaining six will be biallelic $\left(\mathrm{A}_{i} \mathrm{~A}_{i} \mathrm{~A}_{j} \mathrm{~A}_{j}\right.$, $\left.\mathrm{A}_{i} \mathrm{~A}_{i} \mathrm{~A}_{k} \mathrm{~A}_{k}, \quad \mathrm{~A}_{i} \mathrm{~A}_{i} \mathrm{~A}_{l} \mathrm{~A}_{l}, \quad \mathrm{~A}_{j} \mathrm{~A}_{j} \mathrm{~A}_{k} \mathrm{~A}_{k}, \mathrm{~A}_{j} \mathrm{~A}_{j} \mathrm{~A}_{l} \mathrm{~A}_{l}, \quad \mathrm{~A}_{k} \mathrm{~A}_{k} \mathrm{~A}_{l} \mathrm{~A}_{l}\right)$, each with the probability of $1 / 18$. These probabilities then lead to the offspring probabilities, given in Table 2 also for the case of dominance.

For diploids, the probabilities of dominant progeny under automixis are 1,5/6 and 0 for parent genotypes AA, Aa and aa respectively. Probabilities of dominant progeny under selfing are 1, 3/4 and 0 , respectively, whereas those for outcrossing are $1,1-q / 2$ and $1-q$, respectively.

In the estimation procedure below, we need to specify the probabilities of progeny, given the parent genotype, for an arbitrary combination of mating event probabilities. Denote the probabilities given in Table 2 with four arrays, $T, S, U$ and $A$ corresponding to outcrossing, selfing, automixis and apomixis, and each array is indexed by two subscripts, corresponding to parent genotype and progeny phenotype, respectively. With $t, s$, $u$ and $a$ being the rates of outcrossing, selfing, automixis and apomixis in the population $(t+s+u+a=1)$, the probability that a progeny is a dominant phenotype, given parent $j$, is the mixture

$$
P_{j 1}=t T_{j 1}+s S_{j 1}+u U_{j 1}+a A_{j 1}
$$

and likewise with the subscript ' 2 ' for the recessive phenotype.

Now, to estimate the rates of selfing, outcrossing, automixis and apomixis, we require several progeny to be collected from each of several parents, then genotyped. The progeny of a mother is termed a 'progeny array'. To analyze this progeny array data, the following assumptions are made: independence of mating events among progeny, constant gene frequencies, and constant probabilities of mating events. There are two major steps for progeny array analysis: inference of maternal parentage, and estimation of mating frequencies given the parentage.

In the first step, the genotype of the maternal parent is inferred probabilistically from the progeny array as follows (cf. Ritland, 1986). If in family $i, N_{1}$ dominant offspring are observed and $N_{2}$ recessive offspring are observed, the likelihood of the array of phenotypes, is

$$
\begin{aligned}
L_{i j}= & \left(t T_{j 1}+s S_{j 1}+u U_{j 1}+a A_{j 1}\right)^{N_{1}}\left(t T_{j 2}+s S_{j 2}+u U_{j 2}\right. \\
& \left.+a A_{j 2}\right)^{N_{2}}
\end{aligned}
$$

The probability of the array across all possible parents is the sum of the likelihoods over alternative parent genotypes, weighted by the frequency of parent genotypes in the population

$$
L_{i}=\sum_{j} f_{j} L_{i j}
$$

where we assume $f_{j}=(1-q)^{4}, 4 q(1-q)^{3}, \quad 6 q^{2}(1-q)^{2}$, $4 q^{3}(1-q)$, and $q^{4}$ for genotypes AAAA, AAAa, AAaa,
Aaaa and aaaa, respectively. These are approximations that ignore inbreeding. We also assume the pollen gene frequencies equal the population gene frequencies (obtained as the fourth root of the recessive phenotype frequency for tetraploids; the square root for diploids).

In the second step, estimates of mating frequencies are obtained by maximizing the likelihood function of the entire sample. This likelihood is the product of the $L_{i}$ across arrays, and the Newton-Raphson method (see Ritland, 1986) was used to maximize it. The likelihood ratio test was used to detect significant deviations of selfing, outcrossing, automixis and apomixis from zero. A computer program, tsu'nami ( $t, s, u$, 'n a mating inference) implementing this procedure and written in FORTRAN 95 is available from KR upon request.

\section{Evaluation of model properties}

To investigate the theoretical statistical properties of jointly estimating automixis with other mating modes, a FORTRAN 90 program calculated the variance-covariance matix of the estimates of outcrossing rate $t$, selfing rate $s$ and automixis rate $u$. For simplicity, apomixis was omitted as the focus was on the properties of $u$ relative to $s$ and $t$. Also for simplicity, maternal genotype and pollen gene frequency were assumed known. For recessive gene frequencies of $q=0.5$ and $q=0.75$, simulated data sets were generated for sample sizes $N=50,100$ and 200, then the Fisher information matrix (second derivatives of the log-likelihood function) was calculated and inverted to give the variance-covariance matrix, which was then multiplied by $N$ to standardize comparisons. This was replicated 100000 times for each combination of $N$ and $q$ to obtain exact values.

We also evaluated the ability of tsu'nami to recover correct estimates by generating data under a uniform distribution of gene frequencies from 0.2 to 0.8 , for various numbers of families and progeny array sizes, and using tsu'nami to obtain estimates. We considered the four cases of $t=1, s=1, u=1$ and $a=1$. For the diploid population, the same approach was used.

\section{Sampling of arctic Easter daisy populations}

In Canada's Yukon Territory, the perennial and diminutive Easter daisy, T. hookeri, has rare status (Douglas et al, 1981) and is known to comprise disjunct diploid and autopolyploid populations (Thompson and Whitton, unpublished). Diploid plants make viable pollen, whereas polyploid plants are male sterile and putatively apomictic (Beaman, 1957; Thompson and Whitton, unpublished). Apomixis in Townsendia is of the 'Ixeris' type (Beaman, 1957; Nogler, 1984) and is reported to be obligate within the genus (Beaman, 1957). A peculiar meiotic abnormality within the megasporocyte (egg sac precursor) results in the formation of a collapsed restitution nucleus before the development of a $2 \mathrm{~N}$ egg. The segregation pattern of multiple chromosome copies upon division of the restitution nucleus will dictate whether parthenogenesis is automictic (independent segregation) or apomictic (nonindependent segregation leading to an outcome that is genetically equivalent to a mitosis). Pollination is neither required for the development of the embryo nor the endosperm in apomictic Townsendia (Beaman, 1957). 
Plant material was collected from three Yukon populations of T. hookeri: Mile Thirteen $\left(60^{\circ} 59^{\prime} \mathrm{N} 135^{\circ} 10^{\prime} \mathrm{W}\right)$ and Tachäl Dhäl $\left(61^{\circ} 00^{\prime} \mathrm{N} 138^{\circ} 32^{\prime} \mathrm{W}\right)$, both of which consist of tetraploids, and Tantalus Butte $\left(62^{\circ} 07^{\prime} \mathrm{N} 136^{\circ} 15^{\prime} \mathrm{W}\right)$, which is a diploid population. Unopened flower heads were collected from 5-10 plants per population so that anthers could be dissected and male-fertility assessed. Fruits were collected from intact mature capitula from 10 maternal plants for each population. After 3 months of cold treatment, seeds were submerged in $7 \%$ sodium hypochlorite for $15 \mathrm{~s}$, washed in three rinses of distilled water, placed on dampened filter paper, sealed within sterile Petri plates, then imbibed for 3 days in the dark at $4{ }^{\circ} \mathrm{C}$. Germinants were then transferred to $16 \mathrm{~h}$ daylength and room temperature conditions. Seedlings were reared for 5 weeks, until approximately $100 \mathrm{mg}$ of leaf material could be obtained.

\section{AFLP assays}

Genomic DNA was isolated from six progeny from each of the 30 maternal families by the CTAB (hexadecyltrimethylammonium bromide) protocol (Doyle and Doyle, 1987) with the following modifications: volumes were reduced for extraction in $1.5 \mathrm{ml}$ microfuge tubes and sodium metabisulfite $(1 \% \mathrm{w} / \mathrm{v})$ was added to the isolation buffer. Isolated DNA was quantified by a Hoefer DyNAQuant ${ }^{\mathrm{TM}} 200$ fluorometer (San Francisco, CA, USA) according to the manufacturer's directions.

All individuals were AFLP fingerprinted based on the method of Vos et al (1995) with modifications developed by Noyes and Rieseberg (2000). Denatured reaction products were run on an Applied Biosystems 3100 Avant DNA sequencer (Foster City, CA, USA) according to the manufacturer's protocols. Eight primer combinations, $($ EcoACC, EcoACG $) \times($ MeAGC, MseACG, MseACC, MseAAC), were twice screened for three individuals from each of the four populations, and two primer combinations were selected according to the optimality criteria of consistency across all individuals, repeatability for each individual, and level of polymorphism. All individuals were fingerprinted using EcoACC/MseAGC and EcoACG/MseAGC primers then scored for band presence/band absence using Genographer 1.6.0 (Benham, 2001).

\section{Results}

Properties of the estimation procedure

The results of the calculations of the theoretical variancecovariance properties of outcrossing rate $t$, selfing rate $s$ and automixis rate $u$ are given in Table 3. The estimates of selfing and automixis are very strongly negatively correlated, with values of -0.84 to -0.95 , meaning that the statistical information to separate these two components of inbreeding is very slight. This accords with the simulations of estimates, and with the results of the field study (see below), both of which indicated that selfing and automixis were highly confounded. Table 3 also shows that the variances of $s$ and $u$ are comparable, and are much larger than that for $t$, probably because $s$ and $u$ are confounded (if only $s$ and $t$ are estimated, their variances are equal since then $s=1-t)$. In addition, Table 3 shows that when sample sizes are small $(N=50)$, variances of estimates are significantly inflated above
Table 3 Theoretical variances and correlations of estimates per individual sampled when selfing rate, automixis and outcrossing rate are simultaneously estimated, for two frequencies of the recessive marker $q$, and three levels of sample size $(N)$

\begin{tabular}{lrrrrrr}
\hline $\mathrm{N}$ & $\operatorname{Var}(\hat{s})$ & $\operatorname{Var}(\hat{u})$ & $\operatorname{Var}(\hat{t})$ & $\operatorname{Corr}(\hat{s}, \hat{u})$ & $\operatorname{Corr}(\hat{s}, \hat{t})$ & $\operatorname{Corr}(\hat{u}, \hat{t})$ \\
\hline$q=0.5$ & & & & & & \\
50 & 93.4 & 218.4 & 69.64 & -0.84 & 0.34 & -0.78 \\
100 & 62.5 & 72.7 & 7.44 & -0.94 & 0.06 & -0.37 \\
200 & 56.8 & 63.2 & 5.04 & -0.95 & 0.04 & -0.32 \\
$q=0.75$ & & & & & & \\
50 & 59.8 & 58.5 & 1.29 & -0.98 & -0.14 & -0.0009 \\
100 & 55.9 & 54.7 & 1.18 & -0.98 & -0.14 & -0.0023 \\
200 & 54.3 & 53.2 & 1.14 & -0.98 & -0.14 & -0.0041 \\
\hline
\end{tabular}
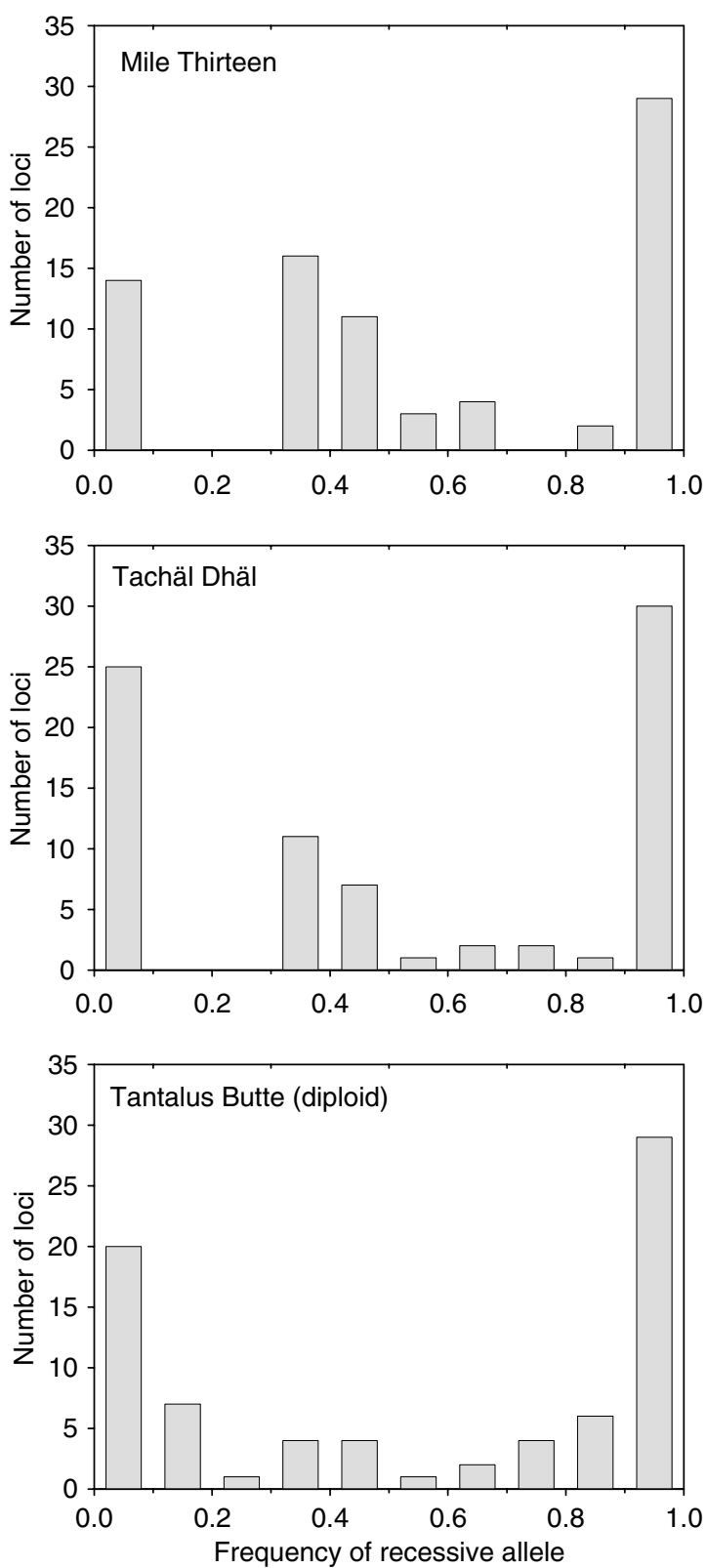

Figure 1 Gene frequency distribution for AFLP markers from one diploid (Tantalus Butte) and two tetraploid (Mile Thirteen and Tachäl Dhäl) arctic populations of the Easter daisy, T. hookeri. 
their asymptotic values (represented by $N=200$ ), particularly when the recessive allele is of lower frequency.

Simulated data indicated that tsu'nami correctly estimated the approximate true values when either selfing or automixis were omitted from the model. However, these simulations indicated that selfing and automixis are difficult to distinguish, in accordance with the results of Table 3 . In the simulations, even with large sample sizes (20 families of size 10, and 1000 loci), data sets consisting of pure selfers gave estimates of about $50 \%$ selfing and $50 \%$ automixis, and conversely, data sets of pure automicts give similar 50-50 proportions of selfing and automixis.

\section{Pollen observations and AFLP results}

Upon dissection, it was readily observed that plants from the Mile Thirteen and Tachäl Dhäl populations failed to produce viable pollen. The stamens of plants from these populations experience early abortion of their anthers. This was confirmed with plants collected in two other field seasons (SL Thompson, personal observations).

A total of 107 AFLP loci were scored for all progeny. Of these 107, 60 loci were from the primer combination EcoACC/MseAGC and 47 were from the primer combination EcoACG/MseAGC. The distribution of gene frequencies in the three study populations, estimated by the procedure above, is given in Figure 1. As a general rule, loci are informative when the gene frequency is between about 0.2 and 0.8 (Ritland, 1986). At frequencies outside of these 'intermediate' values, most mating events involve parents and progeny homozygous for the same alleles, hence different types of matings cannot be distinguished. Figure 1 shows that the Mile Thirteen site had the most informative array of gene frequencies, with 35 of intermediate frequency. Tantalus Butte showed only 16 intermediate frequency loci, while Tachäl Dhäl showed 23 intermediate frequency loci. Thus, we expect the highest precision of estimates in the Mile Thirteen population, and the lowest in the Tantalus Butte population.

\section{Mating patterns in three Easter daisy populations}

Table 4 gives estimates of mating patterns and tests of various hypotheses. For each population, the first line is the case of all four parameters estimated (rates of outcrossing, selfing, automixis and apomixis). The next four lines are hypothesis tests (1) no outcrossing, (2) no selfing, (3) no automixis, and (4) no apomixis. In these tests, three parameters are estimated the fourth set to zero. The last line is the test for (5) no selfing and no automixis; for example, only outcrossing and apomixis was estimated.

When all parameters were simultaneously estimated (line 1, Table 4), all populations showed a predominance of apomixis, from 65 to $72 \%$, and lower levels of selffertilization and automixis, ranging from 3 to $20 \%$. Rates of outcrossing were low in the tetraploid populations, $2-4 \%$, and moderate $(23 \%)$ in the diploid population, Tantalus Butte. To determine the confidence interval for these estimates, log-likelihoods were found across a range of outcrossing rate $t$, for other parameters $(s, u, a)$ jointly estimated (Figure 2). Note the sharp crest of likelihoods at low $t$ for the tetraploid populations, whereas the diploid population (Tantalus Butte) has a much broader crest at a higher $t$, reflecting a higher uncertainty of estimate. To determine whether these differences are significant, we can estimate the confidence interval using the likelihood ratio test. From the point of maximum likelihood $\left(L_{m}\right)$, the point $i$ of lower likelihood at which $-2\left(L_{m}-L_{i}\right)$ equals $3.84\left(\chi^{2}\right.$ with one degree of freedom (1 df)) defines the upper and lower confidence interval (Venzon and Moolgavkar, 1988). The confidence intervals are approximately \pm 0.02 for both tetraploid populations, and \pm 0.06 for the diploid population. Thus, the higher outcrossing rate found in the diploid population is statistically significant.

Table 4 Estimates of outcrossing $t$, selfing $s$, automixis $u$, and apomixis $a$, for each of the three populations, under various hypotheses

\begin{tabular}{|c|c|c|c|c|c|c|}
\hline Model & $t$ & s & $u$ & $a$ & $L n L$ & $-2 \operatorname{Ln}\left(L_{1} / L_{0}\right)$ \\
\hline \multicolumn{7}{|c|}{ Mile thirteen (tetraploid) } \\
\hline Full & 0.02 & 0.06 & 0.20 & 0.72 & -2226.88 & \\
\hline$t=0$ & 0.00 & 0.37 & 0.13 & 0.51 & -2507.05 & 560.34 \\
\hline$s=0$ & 0.02 & 0.00 & 0.29 & 0.69 & -2226.71 & -0.35 \\
\hline$u=0$ & 0.02 & 0.21 & 0.00 & 0.77 & -2227.50 & 1.23 \\
\hline$a=0$ & 0.02 & 0.00 & 0.99 & 0.00 & -2287.06 & 120.36 \\
\hline$s=u=0$ & 0.07 & 0.00 & 0.00 & 0.93 & -2309.58 & 165.39 \\
\hline \multicolumn{7}{|c|}{ Tachäl Dhäl (tetraploid) } \\
\hline Full & 0.04 & 0.16 & 0.15 & 0.65 & -1568.06 & \\
\hline$t=0$ & 0.00 & 0.95 & 0.04 & 0.01 & -1954.38 & 772.65 \\
\hline$s=0$ & 0.04 & 0.00 & 0.37 & 0.59 & -1568.53 & 0.94 \\
\hline$u=0$ & 0.04 & 0.26 & 0.00 & 0.70 & -1567.78 & -0.56 \\
\hline$a=0$ & 0.04 & 0.00 & 0.96 & 0.00 & -1597.42 & 58.72 \\
\hline$s=u=0$ & 0.09 & 0.00 & 0.00 & 0.91 & -1626.28 & 116.45 \\
\hline \multicolumn{7}{|c|}{ Tantalus Butte (diploid) } \\
\hline Full & 0.23 & 0.03 & 0.08 & 0.68 & -1943.71 & \\
\hline$t=0$ & 0.00 & 0.99 & 0.01 & 0.00 & -2526.75 & 1166.08 \\
\hline$s=0$ & 0.21 & 0.00 & 0.12 & 0.67 & -1943.71 & -0.01 \\
\hline$u=0$ & 0.21 & 0.08 & 0.00 & 0.71 & -1943.71 & 0.00 \\
\hline$a=0$ & 0.19 & 0.00 & 0.81 & 0.00 & -1983.01 & 78.59 \\
\hline$s=u=0$ & 0.25 & 0.00 & 0.00 & 0.75 & -1946.38 & 5.34 \\
\hline
\end{tabular}

Also given are log-likelihoods for each model, and likelihood ratio tests for absence of each component of the mating system relative to the full model (negative values are due to slight errors in numerical convergences of likelihood functions). 


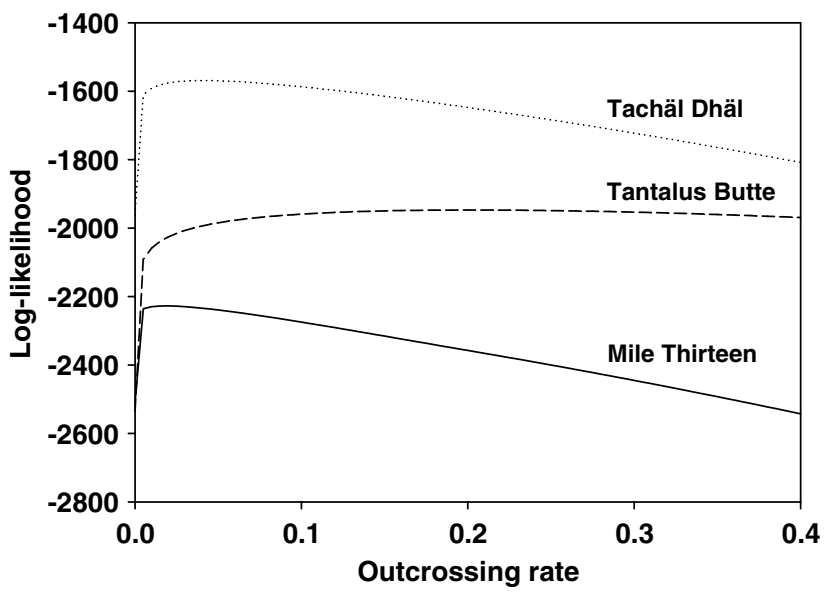

Figure 2 Log-likelihoods across a range of outcrossing rate $t$, for other parameters $(s, u, a)$ jointly estimated. Note the sharp crest of likelihoods at low $t$ for the tetraploid populations, while the diploid population (Tantalus Butte) has a much broader crest at a higher $t$.

Table 4 also gives hypothesis tests about the presence of each mode of mating. The first four likelihood ratio tests in the last column of Table 4 involved comparisons between models with four parameters $(t, s, u$ and $a$ all estimated) $v s$ three parameters (either $t, s, u$ and $a$ set to zero), thus testing for the presence of significant levels of these mating types. In these tests, $-2 \ln \left(L_{1} / L_{0}\right)$, is asympotically distributed as $\chi^{2}$ with $1 \mathrm{df}$, hence values greater than 3.84 are significant at the $95 \%$ level. A final test involved the constraint $u=a=0$ (no apomixis and no automixis), and this test has $2 \mathrm{df}$, with values greater than 6.99 being significant.

In Table 4 , the tests for $t=0$ and $a=0$ were both extremely significant, indicating that outcrossing and apomixis are present in all populations. In contrast, in all three populations, the tests clearly indicate that either automixis or selfing does not exist, as $\chi^{2}$ values were nearly zero. However, the test for $s=u=0$ was highly significant for the tetraploid populations, but not the diploid population (a value of 5.34 was found; 6.99 is needed for significance). Hence, we conclude that either selfing or automixis exists in the tetraploid population, but that the data are not sufficiently informative to distinguish between these two reproductive modes.

\section{Discussion}

Results of the estimation model

Empirical and theoretical treatments of mixed mating systems in plants typically model data as a mixture of selfing and outcrossing, ('mixed mating', reviewed in Goodwillie et al, 2005). Studies of asexuality, however, often employ discrete categories of obligate vs facultative asexuality vs random mating. Studies of outcrossingselfing are rarely combined, or for that matter compared, to studies of sexuality-asexuality (Richards, 1997). This study is the first, to our knowledge, where hypotheses of outcrossing and several types of self-oriented mating have been tested and quantified in tandem.

Our procedure for the inference of mating systems involved assaying progeny arrays for genetic markers.
This is more robust than earlier approaches that estimate population gene frequency and departures from expected heterozygosity under panmixis. The analyses of the segregation of genetic markers among progeny of a common mother are more direct and conclusive (Clegg, 1980).

We have taken the unique approach of testing several mating hypotheses to evaluate the strength of support for each type of mating in relation to other modes of mating. In the two tetraploid populations of the Easter daisy, we detected significant levels of either automixis or selfing, however, we could not ultimately distinguish between the two. Their lack of separation was theoretically confirmed by an extremely negative statistical correlation between the estimates of automixis and selfing in calculations involving the Fisher information index (Table 3). Evidently, the segregation patterns do not sufficiently differ between these two modes of selforiented mating for the case of tetraploidy and dominant markers.

This confounding of the estimates of automixis and selfing can probably be greatly reduced by using codominant markers, and also by a multilocus approach. With codominant markers, the segregation patterns of selfing $v s$ automixis are more distinct. At the multilocus level, 'true' selfing occurs simultaneously at all loci within a single individual, while automixis would occur randomly across loci (with apomixis occurring at the other loci). The joint estimation of automixis with apomixis using these two alternative approaches is an area worth investigating further.

Regardless, through the use of our new method of analysis, we conclude that apomixis is the dominant mode of reproduction in tetraploid populations of the Easter daisy (between 72 and 65\%). The diploid population was also found to reproduce predominantly through apomixis $(68 \%)$, a condition only rarely reported for diploid plants (Nogler, 1984; Asker and Jerling, 1992; Roy, 1995).

\section{Relation to previous studies of apomicts}

The breeding system of putative naturally occurring apomicts has been the subject of several molecular studies involving progeny arrays. Classically, apomixis is detected as fixed heterozygosity among progeny, but the lack of suitable levels of isozyme variation and the complexity of interpreting banding patterns in high-level polyploids have been impediments in many cases (Roy, 1995), whereas the expense in developing and assaying microsatellite markers can be prohibitive for others. Bayer et al (1990) estimated the degree of apomixis vs outcrossing for subpopulations of Antennaria media using codominant isozyme markers. Apomixis rates of 59 and $14 \%$ for $A$. media led to the designation of subpopulations as obligately apomictic and partially apomictic, respectively. Roy (1995) used progeny arrays and isozymes to detect the presence of fixed heterozygosity (species labeled as apomicts) or homozygosity (species labeled as selfers) in six species of Arabis. Ford and Richards (1985) found that in several Taraxacum agamospecies, up to $62 \%$ of offspring had isozyme profiles that differed from their mothers. Based on microsatellite gel patterns, Robertson et al (2004) found that progeny of Sorbus arranensis were all identical to their mothers and denoted 
as obligate, while in S. pseudofennica, $17.5 \%$ of seeds differed in marker phenotype from their mothers. This species was deemed a facultative apomict. Bartish et al (2001) classified Cotoneaster scandinavicus as apomictic based on identical RAPD (randomly amplified polymorphic DNA) profiles among progeny, but classified C. canescens as either selfing or apomictic based on estimates of Jaccard's coefficients of similarity of 0.973 and 0.979 for two accessions. Kollmann et al (2000) detected low levels of AFLP variation among progeny from pseudogamous Rubus armeniacus and $R$. bifrons, leading to their characterization as facultative apomicts. Outcrossing or automixis was suspected in Rubus based on the observation that $14-17 \%$ of seedlings were genetically distinct from the maternal genotype.

If selfing is occurring within arctic polyploid populations of Easter daisies, pollen must be produced at unobservable low levels, as male sterility was observed in natural populations over three field seasons (SL Thompson, personal observation). We favor automixis over selfing, as embryological observations also suggest that automixis is a possibility for polyploid Townsendia (Beaman, 1957).

Automixis has been reported as a possible contributor to high levels of genetic variation within standing populations of apomictic Taraxacum (van der Hulst et al, 2003). Cytological investigations of meioses in natural and synthetic lines of Taraxacum confirm that automixis could be occurring (van Baarlen et al, 2000). The authors also speculate that automixis may be advantageous in the sense that it may limit the accumulation of mutations in parthenogenetic lines, as recessive deleterious mutations can become homozygous and exposed to natural selection. For example, the genotype Aaaa produces aaa progeny $1 / 6$ of the time (Table 2).

The lack of selfing in the diploid Townsendia population suggests that if selfing happens to occur in the tetraploids, which are derived from diploids (albeit perhaps through a triploid intermediate), then it is a derived condition. The transition from diploidy to tetraploidy may either directly cause a shift towards greater selfing, through breakdowns in systems of selfincompatibility (Chawla et al, 1997; Stone, 2002), or indirectly cause an increase in selfing, through selection for increased selfing during the establishment of a neopolyploid undergoing a minority cytotype disadvantage (Ramsey and Schemske, 1998). As well, in tetraploids, it is predicted that inbreeding depression should be reduced (Husband and Schemske, 1997), although this has been rarely tested. If it is reduced, the deleterious effects of selfing should be tolerated to a greater extent. Also, the potentially selfing Townsendia tetraploids exclusively inhabit formerly glaciated regions of the Yukon territory, whereas the nonselfing diploids are located in nonglaciated Beringia. Population recolonization can favor increased selfing, on both demographic grounds (only one individual required to form a new population, Baker, 1955) and ecological grounds (more rapid fixation of successful genotypes within a selfing population, cf. Richards (1997)).

In the two tetraploid Townsendia populations, we found low but highly significant levels of outcrossing (Mile Thirteen: 2\%, Tachäl Dhäl: 4\%). This result is unexpected, as anthers are aborted before the develop- ment of pollen within the polyploid Easter daisy populations in the Yukon territory (Thompson and Whitton, unpublished, Thompson et al unpublished). Plants flower immediately upon spring thaw, before many pollinators become active, and it is not uncommon to find them flowering under snow. It is possible that the low rates of outcrossing we detected are due to genotyping error. Bonin et al (2004) have investigated sources of genotyping error and report rates of $2.6 \%$ for dwarf birch leaves, however, error rates vary with the organism. Possible sources of error include contamination (here reduced through sterile seedling and growth conditions), material used (we used constant tissues of similar age and development, as well as similar amounts of genomic DNA), amplification artifacts, and scoring errors. In future studies, scoring error might be reduced through consistent use of blind samples and automation. At least, genotyping error should not introduce spurious evidence of selfing nor apomixis, as the errors normally introduce nonmaternal alleles.

In summary, this study demonstrates that plantbreeding systems in arctic environments can consist of many components, from outcrossing to the self-oriented modes of self-fertilization, automixis and apomixis. Dominant molecular genetic markers can help identify these modes, but cannot adequately separate automixis from selfing. Rather than thinking of species as strictly following one mode of reproduction (eg obligate apomixis, facultative apomixis) we should focus on the frequencies of various mating strategies within populations, which may ultimately lead to understanding the adaptive significance of these mixtures.

\section{Acknowledgements}

Helpful comments on the manuscript were furnished by Jeannette Whitton, and field support was provided by Bruce Bennett. We thank the reviewers for detailed evaluations that greatly improved the manuscript. A collecting permit for Kluane National Park and a Scientists and Explorers License from the Territorial government of the Yukon are both acknowledged. This research was supported via Natural Science and Engineering Research Council (NSERC) of Canada graduate fellowships to SLT. Fieldwork was supported by a Northern Scientific Training Program grant to SLT.

\section{References}

Asker SE, Jerling L (1992). Apomixis in Plants. CRC Press: Boca Rotan, FL, USA.

Baker HG (1955). Self compatibility and establishment of long distance dispersal. Evolution 9: 337-349.

Bartish IV, Hylm B, Nybom H (2001). RAPD analysis of interspecific relationships in presumably apomictic Cotoneaster species. Euphytica 120: 273-280.

Bayer RJ, Ritland K, Purdy BG (1990). Evidence of partial apomixis in Antennaria media (Asteraceae: Inuleae) detected by the segregation of genetic markers. Am J Bot 77: 10781083.

Beaman JH (1957). The systematics and evolution of Townsendia (Compositae). Contrib Gray Herbarium Harvard Univ 183 $1-151$.

Benham JJ (2001). Genographer. v. 1.6.0 http://hordeum.oscs. montana.edu/genographer. 
Bonin A, Bellemain E, Eidesen PB, Pompanon F, Brochmann C, Taberlet $P$ (2004). How to track and assess genotyping errors in population genetics studies. Mol Ecol 13: 3261-3273.

Brown AHD, Allard RW (1970). Estimation of the mating system in open-pollinated maize populations using isozyme polymorphism. Genetics 66: 133-145.

Chawla B, Bernatzky R, Liang W, Marcotrigiano M (1997). Breakdown of self-incompatibility in tetraploid Lycopersicum peruvianum: inheritance and expression of $S$-related proteins. Theor Applied Genet 95: 992-996.

Clegg MT (1980). Measuring plant mating systems. Bioscience 30: $814-818$.

Cook LM, Soltis PS (2000). Mating systems of diploid and allotetraploid populations of Tragopogon (Asteraceae). II. Artificial populations. Heredity 84: 410-415.

Douglas GW, Argus GW, Dickson HL, Brunton DF (1981). The Rare Vascular Plants of the Yukon. National Museums of Canada: Ottawa, Canada.

Doyle JJ, Doyle JD (1987). A rapid DNA isolation procedure for small quantities of fresh leaf tissue. Phytochem Bull 19: 11-15.

Ford H, Richards AJ (1985). Isozyme variation within and between Taraxacum agamospecies in a single locality. Heredity 55: 289-291.

Goodwillie C, Kalisz S, Eckert CG (2005). The evolutionary enigma of mixed mating systems in plants: occurrence, theoretical explanations and empirical evidence. Ann Rev Ecol Systemat 36: 47-79.

Husband BC, Schemske DW (1997). The effect of inbreeding in diploid and tetraploid populations of Epilobium angustifolium (Onagraceae): implications for the genetic basis of inbreeding depression. Evolution 51: 737-746.

Julier B, Flajoulot S, Barre P, Cardinet G, Santoni S, Huguet T et al (2003). Construction of two genetic linkage maps in cultivated tetraploid alfalfa (Medicago sativa) using microsatellite and AFLP markers. BMC Plant Biol 3: 9-28.

Kollmann J, Steinger T, Roy BA (2000). Evidence of sexuality of European Rubus (Rosaceae) species based on AFLP and allozyme analysis. Am J Bot 87: 1592-1598.

Levin DA (1983). Polyploidy and novelty in flowering plants. Am Natural 122: 1-25.

Mable BK (2004). Polyploidy and self-compatibility: is there an association? New Phytol 162: 803-811.

Mogie M (1986). Automixis: its distribution and status. Biol J Linnean Soc 28: 321-329.

Murawski DA, Fleming TH, Ritland K, Hamrick JL (1994). Mating system in Pachycereus pringlei: an autotetraploid cactus. Heredity 72: 86-94.
Nogler GA (1984). Gametophytic apomixis. In: Johri BM (ed) Embryology of Angiosperms. Springer-Verlag: Berlin. pp 475518.

Noyes RD, Rieseberg LH (2000). Two independent loci control agamospermy (apomixis) in the triploid flowering plant Erigeron annuus. Genetics 155: 379-390.

Otto SP, Whitton J (2000). Polyploid incidence and evolution. Ann Rev Genet 34: 401-437.

Ramsey J, Schemske DW (1998). Pathways, mechanisms, and rates of polyploid formation in flowering plants. Ann Rev Ecol Systemat 29: 467-501.

Richards AJ (1997). Plant Breeding Systems. Chapman and Hall: London.

Ritland K (1986). Joint maximum likelihood estimation of genetic mating structure using open-pollinated progenies. Biometrics 42: 25-43.

Ritland K (1990). A series of FORTRAN programs for estimating plant mating systems. J Heredity 81: 235-237.

Ritland K (2002). Extensions of models for the estimation of mating systems using $n$ independent loci. Heredity 88: 221-228.

Robertson A, Newton AC, Ennos RA (2004). Breeding systems and continuing evolution in the endemic Sorbus taxa on Arran. Heredity 93: 487-495.

Ronfort J (1999). The mutation load under tetrasomic inheritance and its consequences for the evolution of the selfing rate in autotetraploid species. Genet Res 74: 31-42.

Roy BA (1995). The breeding systems of six species of Arabis (Brassicaceae). Am J Bot 82: 869-877.

Stebbins GL (1950). Variation and Evolution in Plants. Columbia University Press: New York.

Stone JL (2002). Molecular mechanisms underlying the breakdown of gametophytic self-incompatibility. Quart Rev Biol 77: $17-32$.

van Baarlen P, van Dijk PJ, Hoekstra RF, de Jong JH (2000). Meiotic rcombination in sexual diploid and apomictic triploid dandelions (Taraxacum officinale L.). Genome 43: 827-835.

van der Hulst RGM, Mes THM, Falque M, Stam P, den Nijs JCM, Bachmann K (2003). Genetic structure of a population sample of apomictic dandelions. Heredity 90: 326-335.

Venzon DJ, Moolgavkar SH (1988). A method for computing profile-likelihood based confidence intervals. Appl Statist 37: 87-94.

Vos P, Hogers R, Bleeker M, Reijans M, van de Lee T (1995). AFLP: a new technique for DNA fingerprinting. Nucleic Acids Res 23: 4407-4414. 